\title{
Comparison of vertical breast displacement between two different sports bra designs
}

\author{
Parunchaya JAMKRAJANG, Weerawat LIMROONGREUNGRAT \\ College of Sports Science and Technology, Mahidol University, Nakhon Pathom, Thailand. \\ Address corresponce to P. Jamkrajang, e-mail: weerawat.lim@mahidol.edu
}

\begin{abstract}
Breast pain during exercise could come from the strain on the breast due to large repetitive breast motion. Proper sports bra should provide not only a support but also reduce breast movement. Since movement in each sport is different, several sport bras are presently designed for each particular sport motion such as walking, jogging and running. However, breast displacement of these sport bra designs has not been investigated particularly in Thailand. The purpose of this study was to compare vertical breast displacement between 3 conditions: no bra (NB), compression bra (CB) and encapsulation (EB). Thirty female collegiate athletes (meanage $=22.33 \pm 2.7 \mathrm{yrs}$, mean $w_{t} 57.3 \pm 5.9 \mathrm{Kg}, \mathrm{BMI}=20.9 \pm 1.4$ ) cup size B75 $-\mathrm{C} 70$ were volunteered in the study. All subjects were required to walk $(3 \mathrm{~km} / \mathrm{hr})$, jog $(6 \mathrm{~km} / \mathrm{hr})$ and run $(9 \mathrm{~km} / \mathrm{hr})$ on the treadmill for 5 minutes under 3 bra conditions. The order of both bra conditions and activities were randomly assigned. Four retro reflective markers were placed on both nipples and on clavicles directly above nipple lines. Eight $100-\mathrm{Hz}$ cameras were used to collect kinematic data. The results showed that no statistically significant differences of vertical breast displacement during walking and jogging between two types of sports bra. However, vertical breast displacement of EB during running was significantly greater than CB. Nevertheless, the displacement between the left and right sides was slightly different on EB while CB was almost symmetrical. In conclusion, CB provides greater support and reduction of vertical breast displacement. However, this design does not provide breast motion independently.
\end{abstract}

Keywords: Kinematics, sports bra, breast motion.

\section{INTRODUCTION}

It has been well documented that sports bra can not only minimize breast displacement but also reduce breast pain and discomfort during excessive movement $(2,3,6)$. Previous studies also suggested that women should select appropriate and comfortable sports bra $(1,3)$. Presently, there are two major types of sports bra design: compression bra (CB) and encapsulation bra (EB). CB is designed to compress the breasts to chest wall while EB is encapsulated the breast separately which allows independent breasts motion.

Several sport bra studies have investigated vertical breast motion by compare either to no bra or daily bra conditions $(3,4,5)$. Mason et al. (3) reported breast displacement between two different sports bra designs during various exercises including walking, jogging and running. It seemed that encapsulation bra reduced vertical breast motion better than compression bra. Nevertheless, this study only conducted on three subjects. Scurr et al. (6) compared breast movement between no bra, everyday bra and sports bra of 15 females running at speed of $2.3 \mathrm{~m} / \mathrm{s}$. The results showed that vertical breast velocity had the strongest relationship to the comfort.

Starr et al. (8) studied vertical breast displacements in two basic design styles of sports bras: compression bra and encapsulation bra for supporting breasts. The participant age was between 23 to 37 years and cup size of breast 32DD, 34D/DD or $36 \mathrm{C} / \mathrm{D}$. They found the participants who wore commercially available compression bra appear higher level of vertical breast displacements. Besides, subjects who wore the prototype sport bra with both compression and encapsulation showed lower levels of vertical breast displacement (8). However, the study investigates in vertical breast movement between $\mathrm{EB}$ and $\mathrm{CB}$ during running various speed were limited. Therefore, the purpose of this study was to compare vertical breast displacement in women with medium breast size between 2 different sports bra designs during different activities including walking, jogging and running. 


\section{MATERIALS AND METHODS}

\section{Participants}

All participants signed an informed consent which was approved by Mahidol University Institution Review Board. Thirty healthy females who had experienced no breast surgery and had not gone through pregnancy within the last year participated in this study. Mean age, weight and height of participants were $22.3 \pm 2.7$ years old, $57.3 \pm 5.9 \mathrm{Kg}$ and $165.1 \pm 6.7 \mathrm{~cm}$, respectively. The same professional bra fitter determined the breast size. There were 12 participants who had breast size B75 and 18 participants who breast size C70.

\section{Experimental procedure}

Four reflective markers were attached on right and left nipples and clavicles. Eight optoelectronic cameras (OptiTrack Inc., USA) were used to record during a static trial and motion at the sampling rate of $100 \mathrm{~Hz}$. The static trails were captured for $2 \mathrm{sec}$. and used as reference positions. Participants were warm-up by walking on a treadmill for 10 minutes. Then, participants were asked to walk or run on a treadmill for 5 minutes at three different speeds of 3 , 6 and $9 \mathrm{~km} / \mathrm{hr}$. The markers during two consecutive foot strikes of each foot were digitized and selected for analysis. All participants were performed under three conditions: no bra (NB), wear compression bra (Nike: Pro Women's Sports bra) and wear encapsulation bra (Wacoal: W027I). The order of the conditions was randomly tested. Raw coordinates were interpolated and filtered using Butterworth with cutoff frequency of $10 \mathrm{~Hz}$. Vertical breast displacement was determine from the difference between vertical coordinate of the nipple during the static no bra trial and each dynamic trial.

\section{Statistical Analysis}

A repeated measure ANOVAs were used to determine the differences of vertical breast displacement between two bra designs $(\mathrm{p}<0.05)$.

\section{RESULTS}

One-way repeated measured ANOVA demonstrated the significance difference between three bra conditions during different activities (Table 1). Post hoc analysis revealed that vertical breast displacement of $C B$ had significantly lesser than EB during jogging and running $(\mathrm{p}<0.05)$.

Relative change of vertical breast displacement between each sport bra and no bra condition was used to determine percent of vertical breast reduction (Figure 1). It was found that sports bra can reduce vertical breast displacement more than $50 \%$ during jogging.

\begin{tabular}{lcccc}
\multicolumn{4}{l}{ Table 1. Vertical breast movements of three bra conditions during different activities (Mean \pm SD). } \\
\hline Activities & Breast vertical displacement $(\mathrm{mm})$ & $\mathrm{NB}$ & $\mathrm{EB}$ & $\mathrm{CB}$ \\
\hline \multirow{4}{*}{ Walking } & Right & $11.7 \pm 3.4$ & $10.9 \pm 3.0$ & $9.7 \pm 3.1$ \\
& Left & $11.2 \pm 4.2$ & $10.3 \pm 2.7$ & $9.9 \pm 2.9$ \\
Jogging & Right & $31.7 \pm 10.4$ & $19.2 \pm 6.8^{*}$ & $14.0 \pm 5.5^{* * *}$ \\
& Left & $28 \pm 12.2$ & $17.8 \pm 5.2^{*}$ & $14.7^{*} \pm 5.7^{*}$, ** $^{* *}$ \\
Running & Right & $42.6 \pm 13.3$ & $23.3 \pm 8.8^{*}$ & $18.9 \pm 5.7^{* * *}$ \\
& Left & $48.8 \pm 12.1$ & $22.8 \pm 7.4^{*}$ & $19.1 \pm 6.4^{* * *}$ \\
\hline
\end{tabular}

Abbreviation: NB - no bra, EB - Encapsulation bra, CB -compression bra

"Significant difference between NB vs EB, NB vs CB $(p<0.05)$. "Significant difference between EB vs CB ( $p<0.05)$.

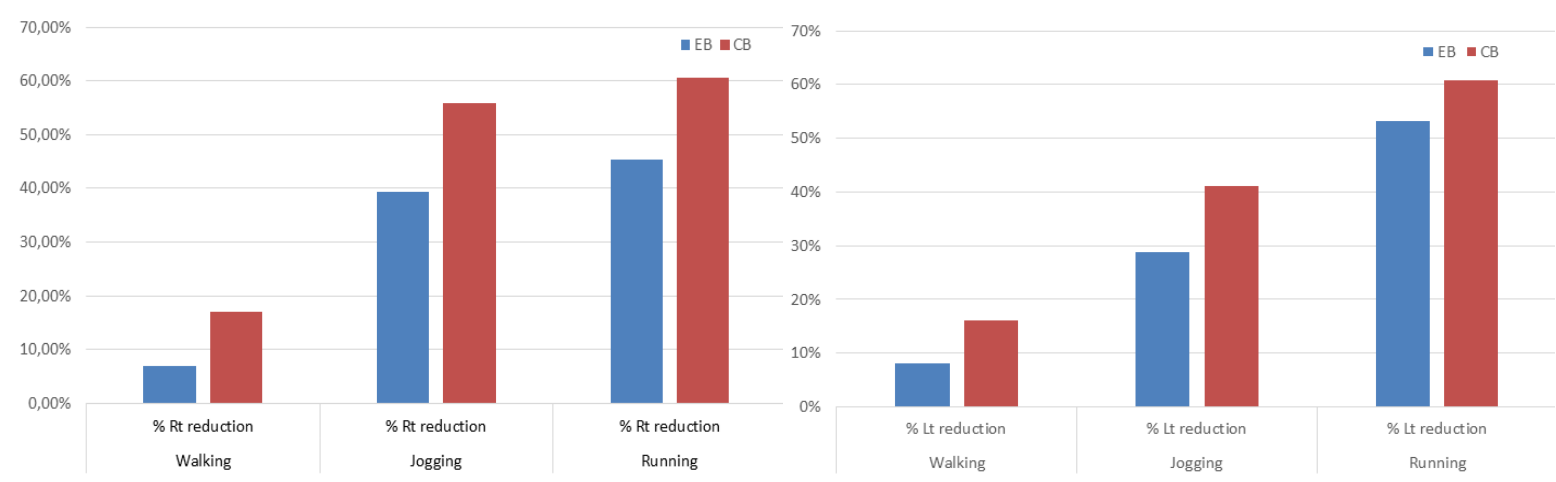

Figure 1. Percent of vertical breast reduction of each sport bra design as compared to no bra condition: Right side (top) and left side (bottom) 


\section{DISCUSSION}

The purpose of this study is to determine vertical breast displacement between the two sport bra designs. Generally, right and left breasts exhibit symmetrical motion in all activities. Vertical breast displacement increased when subjects change their activities from walking to running. When compared to no bra condition, the compression bra and encapsulation bra reduce vertical breast motion about $60 \%$ and $50 \%$, respectively. During walking to running, the breast displacement in the no bra condition increases $40 \%$ whereas the encapsulation and compression bras increase $12 \%$ and $10 \%$, respectively. According to White et al. (10) reported during running the sports bras (EB and $\mathrm{CB}$ ) produced the least vertical bras displacement about $40 \mathrm{~mm}$. both bras, with a reduction in vertical breast displacement of $58.8 \%$ in CB. While, NB produced approximately $90 \mathrm{~mm}$ (2) In addition, there were significant difference of mean vertical breast displacement between $\mathrm{EB}$ and $\mathrm{NB}$, besides $\mathrm{CB}$ and NB were 12.14 and $11.44 \mathrm{~cm}$, respectively.

Moreover, during $\mathrm{CB}$ can reduce breast movement about $60 \%$ that similar to this study during EB which showed reducing breast movement about $45 \%$ and $53 \%$ for right and left respectively. As the results also were similarly of Scurr (4) studied that reported EB could decrease breast movement during running around $50 \%$. Contrary to previous research, CB did not prove more effective at reducing breast movement for small cup size participants; instead, EB was significantly more effective.

In walking, there were no significantly different of breast movements between bra conditions both right and left breast side while, breast vertical displacement during $\mathrm{CB}$ decrease about $16 \%$ and $17 \%$ in left and right side, respectively. Besides, during EB fall around $8 \%$ in left breast side. Moreover, fall around $8 \%$ from the previous study, there was only Mason et al. (3) reported breast movement. However, Mason et al. (3) studied in 3 participants who have cup size12 B, 14B and 14C (75C, 80C and 80D, respectively) obviously that they were bigger than this study. From the previous study showed when speed increases to running the vertical become significantly greater. For breast displacement during $\mathrm{NB}, \mathrm{EB}$ and $\mathrm{CB}$ there were approximately $40-50,23$ and $20 \mathrm{~mm}$., respectively. The result during jogging and running were similar, there were significant difference between NB-EB and NB-CB in both breast sides. Furthermore, there was significant difference of vertical displacement of breast between EB and CB. There was slight different of breast movement between left and right side in all bra conditions. As the result during jogging, the $\mathrm{CB}$ can reduce more breast movement approximately $40 \%-55 \%$ while, the EB can reduce about $30-39 \%$. While, during running, the CB can reduce more breast movement approximately $60 \%$ and the EB can reduce about $45-53 \%$. These results show different from the trend of Mason et al. (3) study that found EB can reduce breast movement better than $\mathrm{CB}$.

In conclusion; compression bra provides greater support and reduction of vertical breast displacement. However, encapsulation bra provides more independent breast motion

\section{Acknowledgments}

The authors would like to thanks Thai Wacoal Public Company for their support.

\section{REFERENCES}

1. Hadi MSA. Sports brassiere: Is it a solution for mastalgia? The Breast Journal, 2000;6:407-9.

2. Jennifer White JS, Wendy Hedger. A Comparison of ThreeDimensional Breast Displacement and Breast Comfort During Overground and Treadmill Running. Journal of Applied Biomechanics, 2011; 27: 47-53.

3. Mason BR, Page KA, Fallon K. An analysis of movement and discomfort of the female breast during exercise and the effects of breast support in three cases. Journal of Science and Medicine in Sport, 1999; 2(2): 134-44.

4. Scurr J. Breast research! An important area of biomechanical research, The Sport and Exercise Scientist, 2007;13:4-5

5. Scurr J, White J, Hedger W. Breast displacement in three dimensions during the walking and running gait cycles. Journal of Applied Biomechanics, 2009; 25(4): 322-9.

6. Scurr JC, White JL, Hedger W. The effect of breast support on the kinematics of the breast during the running gait cycle. Journal of sports sciences, 2010; 28(10):1103-9.

7. Scurr JC, White JL, Hedger W. Supported and unsupported breast displacement in three dimensions across treadmill activity levels. Journal of sports sciences, 2011; 29(1): 55-61.

8. Starr C, Branson D, Shehab R, Farr C, Ownbey S, Swinney J. Biomechanical analysis of a protocol type sports bra. Journal of Textile and Apparel, Technology and management, 2005; 4:1-14

9. Steele K-APaJR. Breast Motion and Sports Brassiere Design. Sports Med. 1999;27(4):205-11.

10. White J, Scurr J, Hedger WA. Comparison of threedimensional breast displacement and breast comfort during overground and treadmill running. Journal of Applied Biomechanics, 2011; 27: 47-53. 